\title{
Savings for retirement under liquidity constraints: a note
}

\author{
Lorenzo Corsini ${ }^{*}$ and Luca Spataro ${ }^{\dagger}$
}

\begin{abstract}
Pension systems often entail some compulsory saving over which individuals have some degree of choice in terms of the pension plan in which to invest. We analyse whether the choice between alternative plans is affected by the presence of liquidity constraints during working life and we prove that the analytical conditions that determine the choice between different plans are the same in the constrained and unconstrained case.

J.E.L. Classification: D52, D91, G11, G23, H55.

Keywords: Choice on pension plans; optimal portfolio composition; incomplete markets; liquidity constraints.
\end{abstract}

\section{Introduction}

Modern pension systems usually entail compulsory savings over which workers have some degree of choice in terms of the pension plan in which to invest. Given the existence of compulsory savings, agents could find it optimal to indebt themselves in order to off-set too large compulsory rates of contribution. Their saving decisions might thus be affected by the presence of incomplete financial markets that prevent them from borrowing the desired amount. Consequentially, liquidity constraints could affect their investment choice. The aim of our work is to analyse what happens to agents' decisions on pension plans when liquidity constraints are binding.

A vast literature has stressed that liquidity constraints affect the amounts saved for retirement (for a review see Magnussen 1994) but not much has been said on how they affect the destination of those savings. Contributions from Dutta et al. (2000), Wagener (2003), Matsen and Thogersen (2004) De Menil et al. (2006), Corsini and Spataro (2011) and Corsini et al. cover the topic of decisions on pension plans but none of them focus on the role of liquidity constraints nor examine in details the emergence of corner solutions.

Our contribution extends a model of optimal choice on pension plans developed by Corsini and Spataro (2011) and, differently from it, focuses on the corner solutions that emerge in the presence of liquidity constraints. Our results show that liquidity constraints do not affect the decisions in terms of the pension plan chosen. The implications of our results are twofold. First, from a methodological point of view, the drop of liquidity constraints from this analysis allows for better analytical tractability without any loss in the

\footnotetext{
* Corresponding author. Dipartimento di Economia e Management, Università di Pisa. Via Ridolfi 10, 56124 Pisa, Italy. Tel: +390502216220 Fax: +39 0502216384. Email: Icorsini@ec.unipi.it.

† Dipartimento di Dipartimento di Economia e Management, Università di Pisa and CHILD. Email: I.spataro@ec.unipi.it.
} 
generality of results. The second implication concerns economic policy: according to our findings, authorities can choose their preferred compulsory contribution rate without worrying that, inducing liquidity constraints, they might somehow bias, through this channel, the choice of individuals' investment plans. In addition, authorities may desire to provide incentives to certain pension plans (for example, to those with higher shares of stocks because this contributes to the development of stock markets or to a higher degree of diversification of individuals' saving portfolios): our result implies that the lessening or tightening of financial constraints (for example, granting easier access to credit services) might not be an effective instrument to achieve this objective.

The work is organized as follows: section 2 develops the basic model with complete financial markets; section 3 introduces incomplete financial markets and explores the role of liquidity constraints and section 4 concludes.

\section{Saving decisions under complete financial markets}

We assume that agents live two periods: in the first they work receiving a wage $w$ and consuming part of their income; in the second they retire, consuming what they have saved. Saving is partly voluntary, cumulated at the risk-free rate $r_{S}$, and partly compulsory since pension system forces individuals to save a fixed contribution rate $\gamma$ in a pension plan of their choice. For sake of simplicity we imagine that only two plans exist: (i) a safe plan $S$ with the risk-free rate of return $r_{S}$ and (ii) a risky plan $R$ whose returns are normally distributed with mean $r_{R}$ and variance $\sigma_{R}^{2}$. Agents choose how much to consume and to save and which pension plan to adopt. Basically, individuals compute their expected indirect utility under the two plans and then choose the one bestowing the highest indirect utility. Thus we first compute the expected indirect utility for each plan: this is done solving a maximization problem with respect to first period consumption. With complete financial markets, an individual under the generic plan $i$ faces the following problem:

1) $\left\{\begin{array}{l}\max _{c_{1}} E\left[U\left(c_{1}, c_{2}\right)\right] \\ \text { s.t. } c_{2} \sim N\left[\left(w-c_{1}\right)\left(1+r_{S}\right)+\gamma w\left(r_{i}-r_{S}\right), \gamma^{2} w^{2} \sigma_{i}^{2}\right]\end{array}\right.$

where $E\left[U\left(c_{1}, c_{2}\right)\right]$ is the expected lifetime utility that depends on consumption in the two periods ( $c_{1}$ and $c_{2}$ respectively). The constraint in Eq. (1) represents the budget constraint: second period consumption is given only by the returns from compulsory and voluntary saving.

A closed form solution can be obtained assuming the following utility function

2) $U=-e^{-a c_{1}}-\rho e^{-a c_{2}}$

where $\rho$ is the rate of time preference and $a$ is the Arrow-Pratt measure of absolute risk aversion.

Following Makarov and Schornick (2010) we assume that $a$ depends on wage with $a=k \cdot w^{-\alpha}$, where $\alpha>0$ represents the elasticity of risk-aversion-to-wage and $k$ is a positive scale factor. This assumption allows us to obtain decreasing-in-income risk aversion, a property that is usually considered the most realistic one. 
Given Eq. (2), we can solve ${ }^{1}$ problem (1) for each plan and obtain the following solution in terms of optimal consumption $c_{1, i}^{*}$ and indirect expected utility $E\left(U_{i}^{*}\right)$ :

3) $c_{1, i}^{*}=\left[\left(1+d_{i}\right) w x-\frac{\log \rho x}{a}\right] \frac{1}{(1+x)}$

4) $E\left(U_{i}^{*}\right)=-(1+x) x e^{-k\left(1+d_{i}\right) w^{1-\alpha} \frac{x}{1+x}} \rho^{\frac{1}{1+x}}$

where $x=1+r_{S}, d_{i}=\gamma \frac{r_{i}-r_{S}-\gamma k w^{1-\alpha} \sigma_{i}^{2} / 2}{x}$ and clearly $d_{S}=0$.

Individuals choose plan $R$ if $E\left(U_{R}^{*}\right)>E\left(U_{S}^{*}\right)$ and according to Eq. (4) this inequality is verified if and only if

5) $d_{R}=\gamma \frac{r_{R}-r_{S}-\gamma \cdot k \cdot w^{1-\alpha} \cdot \sigma_{R}^{2} / 2}{x}>0$

thus the sign of $d_{R}$ determines agents' decisions in terms of pension plans. However, this result is obtained in the absence of liquidity constraints: in the next section we explore the case of liquidity constraints.

\section{The role of liquidity constraints}

If individuals cannot borrow during their working period, the problem (1) can be restated as

6) $\left\{\begin{array}{l}\max _{c_{1}} E\left[U\left(c_{1}, c_{2}\right)\right] \\ \text { s.t. } c_{2} \sim N\left[\left(w-c_{1}\right)\left(1+r_{S}\right)+\gamma w\left(r_{i}-r_{S}\right), \gamma^{2} w^{2} \sigma_{i}^{2}\right] \\ \text { s.t. } c_{1} \leq(1-\gamma) \cdot w\end{array}\right.$

where the second constraint represents the non-borrowing condition and implies that first period consumption cannot exceed net income and that Eqs. (3) and (4) represent now the inner solution of the problem. In particular (6) has an inner solution for

7) $\gamma \leq\left(1+\frac{\log \rho x}{k w^{1-\alpha}}\right) \frac{1}{1+x}-d_{i} \frac{x}{1+x}$.

When the above condition does not hold, constraints become binding. Condition (7) depends on the plan chosen so that constraints might be binding under a plan but not under the other. For plan $S$, we have $d_{s}=0$ and Eq. (7) becomes:

\footnotetext{
${ }^{1}$ Consider that, for any variable $z_{j}$ distributed normally with mean $z$ and variance $\sigma_{z}^{2}$ we have $E\left(e^{-a z_{j}}\right)=e^{-a\left(z-a \sigma_{z}^{2} / 2\right)}$. See Varian (1992) for details.
} 

8) $\gamma \leq\left(1+\frac{\log \rho x}{k w^{1-\alpha}}\right) \frac{1}{1+x}$.

For plan $R$ we can insert (5) in (7) and obtain the following:

9) $k w^{1-\alpha} \sigma^{2} \gamma^{2} / 2-\left(2+r_{R}\right) \gamma+\left(1+\frac{\log \rho x}{k w^{1-\alpha}}\right) \geq 0$.

The above is a second order equation in $\gamma$ whose roots are $\gamma_{1,2}=\frac{2+r_{R} \pm \sqrt{\left(2+r_{R}\right)^{2}-4 \sigma^{2}\left(k w^{1-\alpha}+\log \rho x\right)}}{k w^{1-\alpha} \sigma^{2}}$.

Exploiting conditions (8) and (9) we draw in Fig. 1 the couples $(w, \gamma)$ for which optimal consumption in period one is exactly equal to income. We depict three possible cases depending on the value of the parameter $\alpha$. Curve $S$ represents the safe plan and is obtained from condition (8) so that, above curve $S$, liquidity constraints are binding under the plan $S$. Curves $R$ and $R^{\prime}$ represent the risky plan and are obtained from condition (9) so that within these two curves liquidity constraints are binding under plan $R$. Curve $D$ represents the couples $(w, \gamma)$ for which $d_{R}=0$ : above it we have $d_{R}<0$ while below it we have $d_{R}>0$.

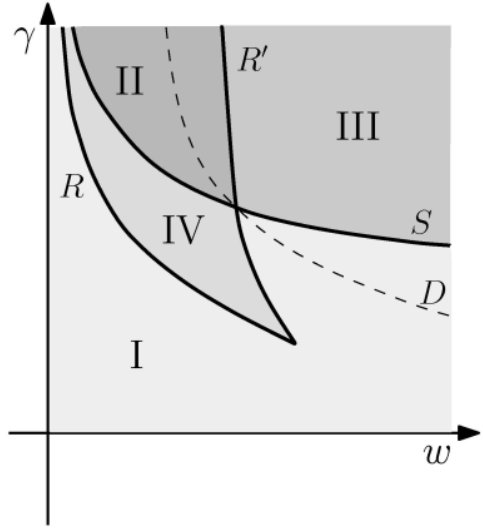

$\alpha<1$

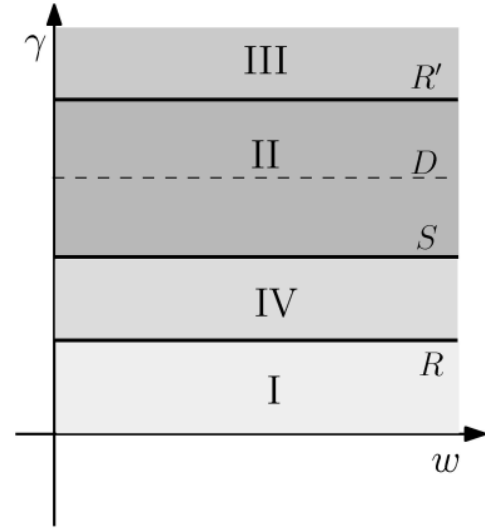

$\alpha=1$

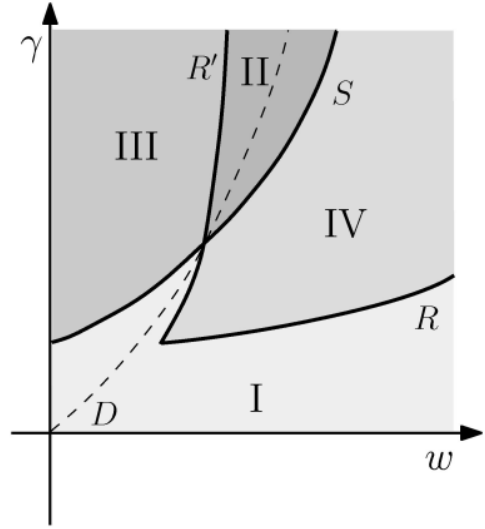

$\alpha>1$

Fig. 1: Regions where constraints are binding

Curves $S, R$ and $R^{\prime}$ define four regions: in region I constraints are not binding for either plans; in region II constraints are binding under both plans; in region III constraints are binding only under plan $S$ and, in region IV, constraints are binding only under plan $R$. Note that region III completely lies above curve $D$ and thus within this region we have $d_{R}<0$, while for region IV the reverse is true.

Whenever the system is outside region I, condition (7) does not hold for at least a plan and, therefore, optimal consumption and indirect utilities are no longer described by Eqs. (3) and (4) but instead the following corner solutions ${ }^{2}$ emerge (the $C$ index denotes the solution when constraints are binding):

\footnotetext{
2 To obtain (12) consider that $E\left(U_{R}^{C}\right)=-e^{-k(1-\gamma) w^{1-\alpha}}-\rho^{\frac{1}{1+x}} e^{-k w^{-\alpha}\left[w\left(1+r_{R}\right)-k w^{-\alpha}\left(w \gamma \sigma_{R}\right)^{2} / 2\right]}$ and $w\left(1+r_{R}\right)-k w^{-\alpha}\left(w \gamma \sigma_{R}\right)^{2} / 2=w x\left(d_{R}+\gamma\right)$.
} 
10) $c_{1, i}^{C}=(1-\gamma) w \forall i$

11) $E\left(U_{S}^{C}\right)=-e^{-k(1-\gamma) w^{1-\alpha}}-\rho e^{-k \gamma \alpha w^{1-\alpha}}$

12) $E\left(U_{R}^{C}\right)=-e^{-k(1-\gamma) w^{1-\alpha}}-\rho e^{-k w^{1-\alpha} x\left(d_{R}+\gamma\right)}$.

The above corner solutions show that, in line with previous literature, the amount saved is affected by liquidity constraints. However, it is still arguable whether such constraints affect the decision on the destination of the compulsory savings. To assess this point we present now three lemmas that characterize individuals' decisions on pension plans when constraints are potentially binding. We will then use the lemmas to formulate a proposition that fully describes individuals' decision on pension plans.

LEMMA 1. If constraints are binding under both the $S$ and $R$ plans, then $R(S)$ is chosen if and only if $d_{R}>(\leq) 0$.

PROOF. If constraints are binding under both plans, then plan $R$ is chosen if and only if $E\left(U_{R}^{C}\right)-E\left(U_{S}^{C}\right)>0$. Starting from Eqs. (11) and (12) and going through computations, we have: $E\left(U_{R}^{C}\right)-E\left(U_{S}^{C}\right)=\rho\left(1-e^{-k w^{1-\alpha} x d_{R}}\right) e^{-k w^{1-\alpha} \gamma x}$. The latter is greater than zero if and only if $d_{R}>0$.

Lemma 1 tells us that if constraints are binding under both plans, then individuals' choice is still univocally determined by the value of $d_{R}$. From a graphical point of view, it tells us that if parameters are such that an individual lies within region II of Fig. 1, then being below (above) Curve D implies the choice of plan $R(S)$ and vice-versa. The economic rationale is that, if constraints are binding under both plans, consumption in period one is clearly the same: thus the chosen plan is the one that bestows higher expected utility in period two, which is univocally determined by the sign of $d_{R}$.

LEMMA 2. If constraints are binding under $S(R)$ plan but not under $R(S)$ plan, then we necessarily have $d_{R}<(>) 0$.

PROOF. If constrains are binding only under the $S(R)$ then condition (7) must be false (true) for $i=S$ but true (false) for $i=R$. Given condition (7), this happens only if $\left(1+\frac{\log \rho x}{k w^{1-\alpha}}\right) \frac{1}{1+x}>(<)\left(1+\frac{\log \rho x}{k w^{1-\alpha}}\right) \frac{1}{1+x}-d_{R} \frac{x}{1+x} \quad$ which can only be true if $d_{R}<(>) 0$.

Graphically, this means that region III (IV) in Fig. 1 is necessarily above (below) Curve D. The economic mechanism behind this result rests on the fact that individuals select, on the base of the sign of $d_{R}$, the plan that implies the higher expect wealth and thus under the chosen plan, given normality of consumption, consumption must be higher in both periods: therefore, it is not possible that an individual is constrained under the non-chosen plan but not under the chosen one.

LEMMA 3. If constraints are binding under the $S(R)$ plan but not under the $R(S)$ plan, then $d_{R} \leq(>) 0$ implies the choice of $S(R)$.

PROOF. If constraints are binding only under plan $S$, then the indirect expected utilities are: 
13) $E\left(U_{S}^{C}\right)=-e^{-k(1-\gamma) w^{1-\alpha}}-\rho e^{-k \gamma 2 w^{1-\alpha}}$

and

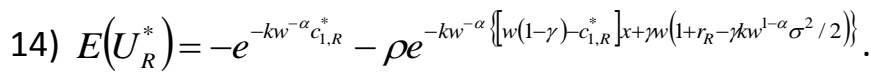

We start comparing the first term of $E\left(U_{S}^{C}\right)$ as given by (13) with the first term of $E\left(U_{R}^{*}\right)$ as given by (14): since by assumption the constraint is not binding under scheme $R$, it must be $c_{1, R}^{*}<(1-\gamma) w$ and thus we have $-e^{-k(1-\gamma) w^{1-\alpha}}>-e^{-k w^{-\alpha} c_{1, R}^{*}}$, that is, the first term of $E\left(U_{S}^{C}\right)$ is always larger than the first term of $E\left(U_{R}^{*}\right)$. We now compute the difference $D$ between the second term of $E\left(U_{S}^{C}\right)$, as given in Eq. (13), and the second term of $E\left(U_{R}^{*}\right)$ :

15) $D=-\rho e^{-k \gamma 2 w^{1-\alpha}}+\rho e^{\left.-k w^{-\alpha}\left\{w(1-\gamma)-c_{1, R}^{*}\right] x+\gamma w\left(1+r_{R}-\gamma k w^{1-\alpha} \sigma^{2} / 2\right)\right\}}$

and inserting (3) in the above and rearranging we obtain

16) $D=-\rho\left[e^{-k \gamma x w^{1-\alpha}}-e^{-k w^{1-\alpha} \frac{x}{1+x}\left(1+d_{R}+\frac{\log \rho}{k w^{1}-\alpha}\right)}\right]$.

The above is positive for $\gamma>\frac{1}{1+x}\left(1+d_{R}+\frac{\log \rho x}{k w^{1-\alpha}}\right)$. Since by assumption constraints are binding under plan $\mathrm{S}$, we know from Eq. (8) that $\gamma>\left(1+\frac{\log \rho x}{k w^{1-\alpha}}\right) \frac{1}{1+x}$ and therefore, whenever $d_{R} \leq 0$ then $\gamma>\frac{1}{1+x}\left(1+d_{R}+\frac{\log \rho x}{k w^{1-\alpha}}\right)$. Thus $d_{R} \leq 0$ implies that $\mathrm{D}>0$, i.e. the second term of $E\left(U_{S}^{C}\right)$ is larger than the second term of $E\left(U_{R}^{*}\right)$.

Since the first term of $E\left(U_{S}^{C}\right)$ is always larger than the first term of $E\left(U_{R}^{*}\right)$ and since $d_{R} \leq 0$ implies that second term of $E\left(U_{S}^{C}\right)$ is larger than the second term of $E\left(U_{R}^{*}\right)$, it follows that $d_{R} \leq 0$ implies that $E\left(U_{S}^{C}\right) \geq E\left(U_{R}^{*}\right)$. The same arguments can be used to prove the case where constraints are binding under the $R$ plan but not under the $S$ plan.

From a graphical point of view, Lemma 3 tells that, within region III and region IV of Fig. 1, being below (above) Curve $\mathrm{D}$ implies the choice of plan $R(S)$. The economic intuition behind this result is that the presence of binding constraints under only a plan is exactly due to the fact that the particular plan implies higher expected wealth and consumption and is, therefore, the chosen plan.

From the three lemmas the following proposition descends

PROPOSITION 1. When individuals face liquidity constraints, they chose plan $R(S)$ if and only if $d_{R}>(\leq) 0$.

PROOF. If liquidity constraints are present, they can be binding: (i) under neither plan, (ii) under both plans, (iii) under plan $S$ only or (iv) under plan $R$ only. In case (i) we obtain inner solutions and we know from Eq. 
(5) that individuals choose the plan $R(S)$ if and only if $d_{R}>(\leq) 0$. In case (ii) we know from Lemma 1 that individuals choose plan $R(S)$ if and only if $d_{R}>(\leq) 0$. In case (iii) we know from Lemma 2 that $d_{R}$ is necessarily negative and thus, from Lemma 3 , plan $S$ is chosen. In case (iv) we know from Lemma 2 that $d_{R}$ is necessarily positive and thus, from Lemma 3 , plan $R$ is chosen. Therefore in all possible cases the pension plan $R(S)$ is chosen if and only if $d_{R}>(\leq) 0$

Proposition 1 basically combines together all the possible combinations in terms of plans and liquidity constraints (that is, all the regions within Fig. 1) and it shows that, in all the possible combinations, the presence of liquidity constraints does not affect the decision on which retirement plan to invest into and this is true even when constraints are actually binding. From a policy point of view, this result shows that, if the authorities desire to provide incentives to certain investment plans, they should target the determinants of the variable $d_{R}$ rather than lessen or tighten financial constraints.

\section{Conclusions}

Our analysis explores investment choices on retirement savings when liquidity constraints are binding. We show that liquidity constraints affect decisions on how much to save for retirement but they do not affect the destination of compulsory savings. In fact we prove that the very conditions for which constraints are binding also guarantee that the choice on pension plans remains the same as in the unconstrained case.

\section{Acknowledgments}

We thank an anonymous referee and the Editor (Roberto Serrano) for their useful comments and suggestions. The usual disclaimer applies.

\section{References}

Corsini, L. and Spataro, L., 2011. Optimal decisions on pension plans in the presence of financial literacy costs and income inequalities. MPRA Paper 30946. University Library of Munich, Germany.

Corsini, L., Pacini, P.M. and Spataro, L., 2012. Workers' Choice on Pension Schemes: A Theoretical Model and an Application to the Italian Second Pillar Reform, Public Finance Review 40 (2), 207-239.

De Menil, G., Murtin, F. and Sheshinski, E., 2006. Planning for the optimal mix of paygo tax and funded savings. Journal of Pension Economics and Finance 5 (1), 1-25.

Dutta, J., Kapur, S. and Orszag, J.M., 2000. A portfolio approach to the optimal funding of pensions. Economics Letters 69 (2), 201-206.

Magnussen, A.K., 1994. Old-age pensions, retirement behaviour and personal saving: a discussion of the literature. Social and Economic Studies 87. Statistics Norway, Oslo-Kongsvinger. 
Makarov, D. and Schornick, A.V., 2010. A note on wealth effect under CARA utility, Finance Research Letters $7(3), 170-177$.

Matsen E. and Thogersen, O., 2004. Designing social security - a portfolio choice approach, European Economic Review 48 (4), 883-904.

Wagener, A., 2003. Pensions as a portfolio problem: fixed contribution rates vs. fixed replacement rates reconsidered, Journal of Population Economics 16 (1), 111-134.

Varian, H.R., 1992. Microeconomic Analysis, $3^{\text {rd }}$ edition, New York: Norton.

FIG. 1 CAPTION: Regions where constraints are binding 